\title{
Enterocolitis necrotizante fulminante en un bebé colodión. Caso clínico
}

\author{
YOLANDA MARTÍNEZ-CRIADO ${ }^{1}$, ANA MILLÁN L. ${ }^{1}$, \\ ANTONIO PAVÓN D. ${ }^{2}$, JUAN CARLOS DE AGUSTÍN A. ${ }^{1}$ \\ 1. Servicio de Cirugía Pediátrica, Hospital Infantil Virgen Del Rocío, Sevilla, España. \\ 2. Servicio de Neonatología, Hospital Infantil Virgen Del Rocío, Sevilla, España.
}

\begin{abstract}
Fulminant necrotizing enterocolitis in a collodion baby. A case report

Introduction: Collodion baby is a rare neonatal ichthyosis. The leathery, hard and yellow skin that covers the body is an ineffective barrier that predisposes to dehydration, heat loss and infections. A better management of these patients has been shown to increase survival, but the comorbidity requires strict and cautious monitoring. Objective: To describe a case of fulminant enterocolitis in a collodion infant in order to help to identify early this pathology. Case report: A 34-week newborn with collodion membrane was admitted to the intensive care unit with severe respiratory distress. The baby was maintained in an incubator with humidity, applying emollients and skin and eye lubrication. Broad-spectrum antibiotic treatment was initiated. The patient presented feeding intolerance on the fifth day of life, developing a fulminant enterocolitis resulting in death. Conclusion: The necrotizing enterocolitis associated with a collodion baby has not been described in the literature, causing a delay in diagnosis and contributing to a fulminant course that can lead to death. Keeping in mind this rare comorbidity may facilitate a timely management.
\end{abstract}

(Key words: Collodion baby, necrotizing enterocolitis, ichthyosis, dehydration).

Rev Chil Pediatr 2013; 84 (4): 429-433

\section{RESUMEN}

Introducción: El bebé colodión es una ictiosis neonatal muy poco frecuente. La piel apergaminada, amarilla y dura que recubre el cuerpo resulta ser una barrera ineficaz, que predispone a la deshidratación, pérdida de calor e infecciones. El mejor manejo de estos pacientes ha permitido aumentar su supervivencia, pero su comorbilidad requiere de un seguimiento estricto y cauteloso. Objetivo: Describir un caso de enterocolitis fulminante desarrollada en un bebé colodión, con el fin de ayudar a la identificación más precoz de esta patología. Caso clínico: Recién nacido de 34 semanas, con membrana colodión, que ingresa en la unidad de cuidados intensivo con dificultad respiratoria severa. Se mantiene en una incubadora con humedad, aplicando emolientes y lubricación cutánea y oftalmológica. Se inició tratamiento antibiótico de amplio espectro. El quinto día de vida,

Recibido el 9 de julio de 2012, devuelto para corregir el 19 de diciembre de 2012, segunda versión 15 de enero de 2013, tercera versión 26 de febrero de 2013, aceptado para publicación el 1 de julio de 2013.

Todos los autores han participado en el caso. No existe ningún conflicto ético ni de intereses.

Los padres dieron la autorización para la divulgación científica del mismo.

Este trabajo cumple con los requisitos sobre consentimiento /asentimiento informado, comité de ética, financiamiento, estudios animales y sobre la ausencia de conflictos de intereses según corresponda.

Correspondencia a:

Yolanda Martínez-Criado

E-mail: yoli84mc@hotmail.com 
comenzó con intolerancia a la alimentación, desarrollándose una enterocolitis fulminante que le ocasionó el fallecimiento. Conclusiones: La asociación con enterocolitis necrosante en un bebé colodión no ha sido descrita en la literatura, por lo que, ocasiona una demora diagnóstica y esto contribuye a una evolución fulminante que puede ocasionar la muerte. El tener presente esta infrecuente comorbilidad podrá favorecer su pesquisa para el manejo oportuno.

(Palabras clave: Bebé colodión, enterocolitis necrosante, ictiosis, deshidratación).

Rev Chil Pediatr 2013; 84 (4): 429-433

\section{Introducción}

El bebé colodión es la manifestación fenotípica de las ictiosis congénitas más rara y grave, que se manifiesta en el período neonatal. Existen aproximadamente 270 casos descritos en la literatura. La mayoría evolucionan a una ictiosis laminar cuya herencia es autosómica recesiva, causada por una mutación en el gen de la transglutaminasa 1 (TGM1) que codifica para un transportador de lípidos de los queratinocitos, lo que origina un exceso de queratinización epidérmica y defecto de descamación. Al nacimiento, se caracteriza por una piel apergaminada brillante y dura, con tendencia a fisurarse en las zonas de flexión. Provoca ectropión y eclabium en los casos más graves. Esta cubierta dificulta la alimentación, los movimientos respiratorios y articulares durante los primeros días de vida. La barrera cutánea resulta ineficaz, produciéndose alteraciones en la termorregulación, pérdida de agua con tendencia a la deshidratación hipernatrémica e incrementa el riesgo el riesgo de infección ${ }^{1-3}$. El diagnóstico inicial es clínico, ya que las biopsias de piel no nos permiten diferenciar entre las distintas enfermedades que causan membrana colodión. El estudio genético es fundamental para confirmar el desarrollo de una ictiosis laminar en el futuro, y para facilitar un consejo genético a la familia.

En los últimos veinte años, el pronóstico de estos pacientes ha mejorado, en parte gracias al mejor manejo en las unidades de cuidados intensivos neonatales. Sin embargo, el riesgo de sepsis, comorbilidad respiratoria y alteraciones hidroelectrolíticos siempre existe ${ }^{2}$.

La asociación de enterocolitis necrosante con ictiosis en harlequín no ha sido descrita en la literatura. El objetivo de este trabajo, es presentar un caso de un bebe colodión que desarrolló una enterocolitis necrosante fulminante que le ocasionó la muerte, para ayudar a la identificación precoz de esta patología en pacientes con membrana colodión.

\section{Caso clínico}

Recién nacido de 34 semanas, sexo femenino, que ingresó en la UCI neonatal por ictiosis congénita y dificultad respiratoria severa que requirió de ventilación mecánica. Madre de 35 años con estudio genético que objetivó la mutación c.877-2A > G del gen TGM1 del cromosoma 14, haciéndola portadora sana de ictiosis laminar congénita. El padre también era portador sano, con la mutación p.Arg142His del gen de la TGM 1. No existía consanguinidad entre los padres. Hermano con ictiosis laminar autosómica recesiva en la actualidad, y membrana colodión al nacimiento que evolucionó favorablemente sin presentar complicaciones añadidas. Parto por cesárea electiva, de inicio espontaneo, con Apgar de 9-9-10.

Al nacer, la antropometría mostró un peso de $2.150 \mathrm{gr}$, talla de $40 \mathrm{~cm}$ y perímetro craneal de $32 \mathrm{~cm}$. En el examen físico el cuerpo entero se encontraba cubierto de una piel gruesa, amarillenta y brillante (membrana colodión), con múltiples fisuras en zonas de flexión y región perineal (figura 1). Severo ectropion bilateral con edema palpebral y eclabium (labios evertidos en "boca de pez"). Manos y pies ligeramente en posición de semiflexión, con uñas normales (figura 2). Motilidad espontánea mínima. Auscultación cardiaca con tonos rítmicos, sin soplos. Auscultación pulmonar con regular entrada de aire bilateral.

El paciente presentaba dificultad respiratoria severa, con resistencia a la expansión de la caja torácica. En la gasometría se detectó 
acidosis respiratoria severa, requiriendo intubación para ventilación convencional en la primera hora de vida ante la imposibilidad de la ventilación no invasiva. A las tres horas de vida se administró una dosis de surfactante mejorando la dificultad respiratoria debida a la inmadurez pulmonar. La auscultación cardiopulmonar no mostró soplos ni alteraciones del ritmo cardiaco. Murmullo vesicular conservado, con crépitos en ambas bases.

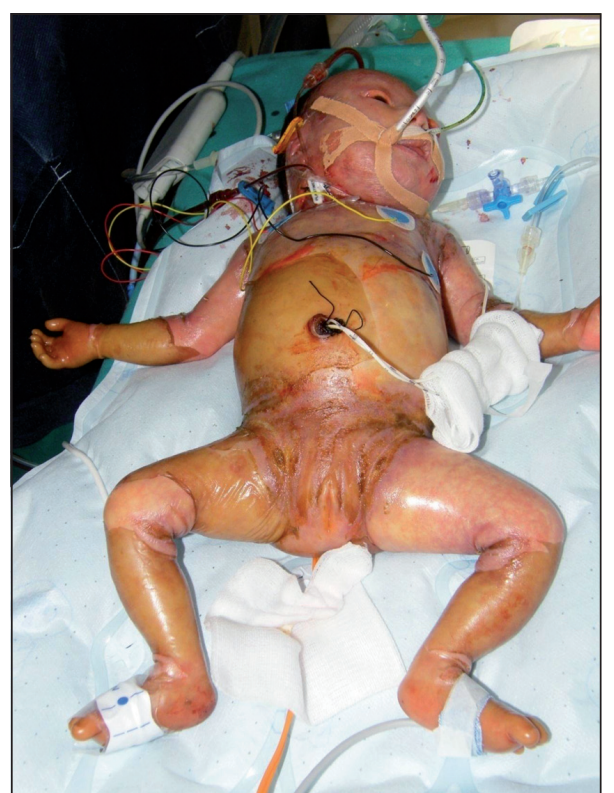

Figura 1. Membrana amarillenta, gruesa, dura y tensa. Obsérvese las fisuras y el inicio de la descamación.
Desde su ingreso, se mantuvo en incubadora con humedad y temperatura elevada, usándose lubricantes y emolientes tópicos con una evolución favorable, comenzando con una descamación dérmica al quinto día de vida. Para el ectoprion, se procedió a lubricación con lágrimas artificiales y una pomada antifúngica.

Desde el ingreso se inició profilaxis antibiótica con ampicilina y gentamicina, al cuarto día llegaron cultivos positivos para Klebsiella pneumoniae, iniciándose tratamiento con carbapenem asociado a antifúngicos ante la persistencia del deterioro clínico.

La alimentación enteral se inició a través de sonda nasogástrica, con buena tolerancia inicial. Al quinto día de vida, comenzó con intolerancia oral, vómitos y distensión abdomi-

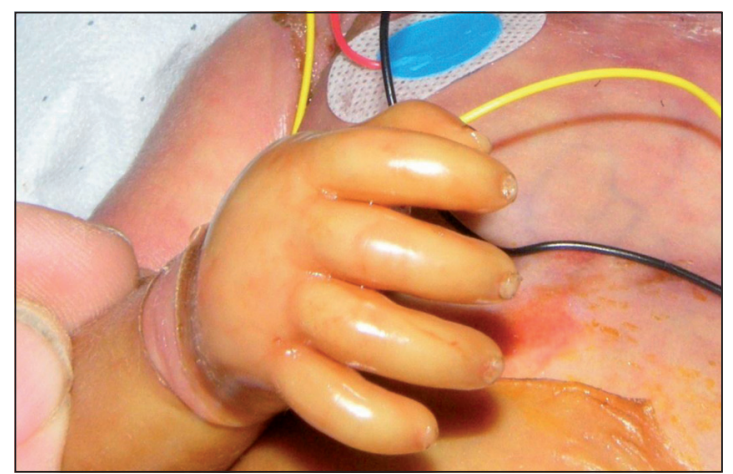

Figura 2. Membrana colodión que provoca contractura articular en posición de semiflexión, dificultando los movimientos espontáneos.
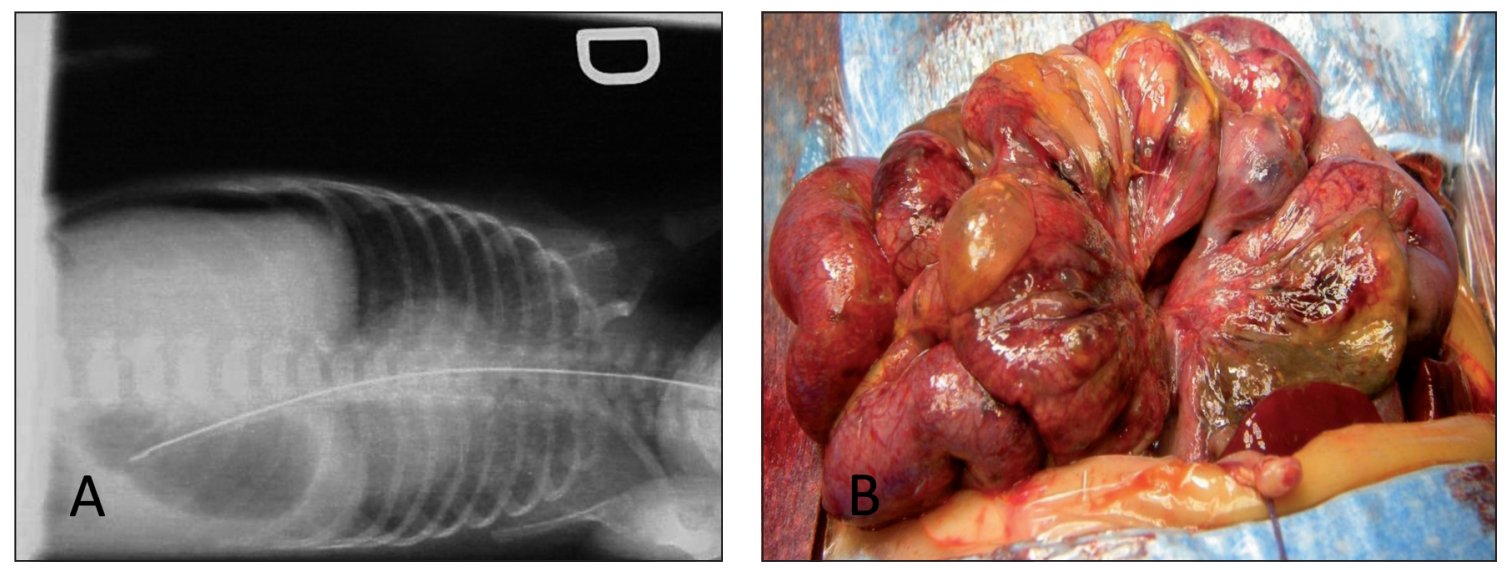

Figura 3. A. Radiografía: neumoperitoneo. B. Intraoperatoria: enterocolitis necrosante fulminante que afectaba a más del $80 \%$ del intestino. 
nal. No se observaron cambios de coloración cutánea debidos a la membrana colodión. Se realizó una radiografía simple de abdomen, en la que se visualizó un neumoperitoneo (figura $3 \mathrm{~A}$ ). Ante los hallazgos se procedió a realizar una laparotomía exploradora urgente, observándose múltiples parches de necrosis y perforaciones intestinales que se suturaron, diagnosticándose intraoperatoriamente enterocolitis necrotizante (figura 3B). En el postoperatorio el paciente estuvo inestable desde el punto de vista hemodinámico, precisando de soporte ionotrópico y ventilación de alta frecuencia. Nueve días más tarde se realizó laparotomía exploradora o "second look" ante la mala evolución clínica y el empeoramiento analítico, con plaquetopenia y anemia severa que precisaron de transfusiones. En esta revisión se encontraron nuevas perforaciones con una necrosis intestinal total. El diagnóstico intraoperatorio fue de una enterocololitis necrosante fulminante.

A los 17 días de vida falleció. En los resultados de la autopsia, se diagnosticó una neumonía bilateral por Stenotrophomonas maltophilia y enterocolitis necrotizante.

\section{Discusión}

El bebé colodión representa una dificultad en el tratamiento, no sólo por la predisposición a la infección y la deshidratación hipernatrémica debidos a la barrera dérmica ineficaz, sino también por los problemas de la prematuridad asociada como es nuestro caso ${ }^{1}$.

El manejo inicial de un bebé colodión incluye una humidificación de la incubadora, control de la temperatura, soportes nutricionales, cuidados de la piel y los ojos, control del dolor, soporte respiratorio y prevención de las infecciones. Las pérdidas cutáneas de agua y calor por el aumento de la permeabilidad cutánea, deben controlarse humidificando y aumentando la temperatura de la incubadora, así como aplicando emolientes tópicos. La dificultad para realizar los movimientos deglutorios y la inflamación crónica, hace necesario que la alimentación enteral se inicie a través de una sonda nasogástrica en los primeros días y con un $25 \%$ más de calorías que lo que le correspondería para su edad. Para el cuidado cutáneo, se debe aplicar generosamente emolientes varias veces al día, con el objetivo de protegerlo frente a patógenos y disminuir las pérdidas de agua. La aplicación de queratinolíticos tópicos (ácido salicílico, urea) o sistémicos (ácido retinoico) deben de evitarse por la toxicidad asociada, siendo además innecesarios, ya que la descamación suele iniciarse al final de la primera semana ${ }^{2}$.

Es importante el control de dolor que originan las fisuras cutáneas. En muchos casos, las fisuras del tórax dificultan los movimientos respiratorios aumentando el riesgo de complicaciones pulmonares. En nuestro caso, el paciente precisó de ventilación mecánica por dificultad respiratoria severa, y a pesar de un óptimo manejo integral, el desarrollo de una neumonía por un germen multirresistente, Sternotrophomonas maltophilia ${ }^{4}$, fue una de las causas del fallecimiento.

Debido a la complejidad del cuadro se requiere de un manejo multidisciplinario. El ectropión presente en los casos más severos, requiere de un control por un oftalmólogo, siendo necesaria la aplicación de lágrimas artificiales y antibióticos oculares ${ }^{5}$. La contractura articular, especialmente en las manos, requiere de un manejo precoz por un rehabilitador pediátrico. En los casos más graves es necesaria la amputación por gangrena digital distal por traumatólogos infantiles ${ }^{6}$.

La sepsis es un problema muy grave y constante, que puede causar la muerte de estos pacientes. La ineficacia de la barrera cutánea con fisuras profundas, hacen necesario la administración de antibióticos y/o antifúngicos profilácticos de amplio espectro desde el nacimiento.

$\mathrm{Su}$ comorbilidad con enterocolitis necrosante no ha sido descrita en la literatura. El riesgo de infección bacteriana, la prematuridad, la alimentación con fórmula, la hipovolemia por deshidratación y la elevación de los mediadores inflamatorios son los principales mecanismos patogénicos de enterocolitis necrosante ${ }^{7}$. Todos ellos estaban presentes en nuestro bebé colodión, lo quizás pudo influir en una evolución fulminante. Además, en caso de desarro- 
llo de enterocolitis necrosante, la exploración abdominal por la ictiosis se encuentra enmascarada, no siendo posible detectar la distensión, los cambios de coloración o livideces a ese nivel. Sólo la intolerancia a la alimentación y el aumento de las fisuras a nivel abdominal por el aumento de presión ante la distensión de asas, junto con signos de sepsis permitió la sospecha de patología gastrointestinal concomitante. Pero la demora diagnóstica, produjo una necrosis de todo el intestino que originó una perforación, cuyo neumoperitoneo hizo necesario de una laparotomía urgente.

La mortalidad de los bebés colodión ha descendido desde el $50 \%$ en los años 60 hasta el $11 \%$ en los años 80 , debido al avance de la neonatología como al mejor conocimiento y prevención de las complicaciones ${ }^{2}$.

\section{Conclusión}

En el caso que exponemos, a pesar de un cuidado óptimo, el paciente presentó una evolución tórpida por la enterocolitis necrotizante y su asociación con una neumonía bilateral por Stenotrophomonas maltophilia, un germen multirresistente muy virulento de adquisición típica en las unidades de cuidados intensivos neonatales. La asociación de enterocolitis necrosante en un bebé colodión no ha sido des- crita en la literatura, por lo que, ocasiona una demora diagnóstica y esto contribuye a una evolución fulminante que puede ocasionar la muerte. El tener presente esta infrecuente comorbilidad podrá favorecer su pesquisa manejo oportuno.

\section{Referencias}

1.- Harvey HB, Shaw MG, Morrell DS: Perinatal management of harlequin ichthyosis: a case report and literature review. J Perinatol 2010; 30: 66-72.

2.- Theiler M, Mann C, Weibel L: Self-healing collodion baby. J Pediatr 2010; 157: 169-71.

3.- Rajpopat S, Moss C, Mellerio J, et al: Harlequin ichthyosis: a review of clinical and molecular findings in 45 cases. Arch Dermatol 2011; 147: 681-6.

4.- Brooke JS: Stenotrophomonas maltophilia: an emerging global opportunistic pathogen. Clin Microbiol Rev 2012; 25: 2-41.

5.- Bhardwaj U, Phougat A, Dey M, Raut S, Srivastav G, Gupta Y: A rare case of collodion baby with ophthalmic involvement. Nepal J Ophthalmol 2012; 4: 184-6.

6.- Liu RH, Becker B, Gunkel J, Teng J: Rapid improvement in digital ischemia and acral contracture in a collodion baby treated with topical tazarotene. J Drugs Dermatol 2010 Jun; 9 (6): 713-6.

7.- Lambert DK, Christensen RD, Baer VL, et al: Fulminant necrotizing enterocolitis in a multihospital healthcare system. J Perinatol 2012; 32: 194-8. 